\title{
On the Capacity of Cognitive Relay assisted Gaussian Interference Channel
}

\author{
Sriram Sridharan, Sriram Vishwanath, Syed A. Jafar and Shlomo Shamai (Shitz)
}

\begin{abstract}
This paper studies a two source, two destination Gaussian interference channel in the presence of a cognitive relay. The cognitive relay has access to the messages transmitted by both the sources and assists them in communicating the messages successfully to their respective destinations. An achievable rate region for the system is derived by combining the Han-Kobayashi coding scheme for the general interference channel with dirty paper coding. The paper also derives outer bounds on the capacity region and obtains the degrees of freedom of the system.
\end{abstract}

\section{INTRODUCTION}

Practical wireless communication systems are in general interference limited. The interference channel is a well studied problem. However, the capacity region of the two user interference channel is still an open problem (even for the Gaussian case). The capacity region is known only for a few special classes of interference channels such as the Gaussian interference channel with strong interference [1], discrete memoryless interference channel with strong interference [2], a class of deterministic interference channels [3] and a class of discrete additive degraded interference channels [4]. Recently, Etkin et. al. characterized the capacity region of the Gaussian interference channel to within one bit [5]. In other recent works [6][7], the authors derive new outer bounds for the Gaussian interference channels and achieve the sum capacity under certain channel conditions.

Networks with cognitive users are gaining prominence with the development of cognitive radio technology, which is aimed at improving the spectral efficiency and the system performance by designing nodes which can adapt their strategy based on the network setup. Much recent work has been focused on the two user interference channel with a cognitive transmitter [8]-[12]. In this channel setting, one of the transmitters has non-causal access to the message transmitted by the other transmitter. In this paper, we study a two user Gaussian interference channel in the presence of a cognitive relay. This

S. Sridharan and S. Vishwanath are with the Wireless Networking and Communications Group, Department of Electrical and Computer Engineering, University of Texas at Austin, Austin, TX - 78712 (email: sridhara@ece.utexas.edu; sriram@ece.utexas.edu). S. Sridharan and S. Vishwanath are supported in part by National Science Foundation grants NSF CCF-0448181, NSF CCF-0552741, NSF CNS-0615061, and NSF CNS0626903, THECB ARP and the Army Research Office YIP.

S. A. Jafar is with the Department of Electrical and Computer Science, University of California at Irvine, Irvine, CA 96297 (email : syed@eecs.uci.edu).

S. Shamai (Shitz) is with the Department of Electrical Engineering, Technion-Israel Institute of Technology, Technion City, Haifa 32000, Israel (email : sshlomo@ee.technion.ac.il). The work of S. Shamai was supported by the Israel Science Foundation. channel model is different from the one used in [8]-[12] in that, each transmitter has access to only their respective messages. However, we assume that there is a cognitive relay node which has non-causal access to the messages of both the transmitters. This relay node serves only to assist the two transmitters in communicating their messages to their respective receivers. An achievable region for this system is described in [13].

In this paper, we present a new achievable region for the Gaussian interference channel with a cognitive relay. This region is a generalization of the achievable region given in [13]. The coding scheme used in this paper is a combination of the Han-Kobayashi coding scheme for the general interference channel [14] and Costa's dirty paper coding [15]. The HanKobayashi coding scheme was also used for the interference channel with a normal (non cognitive) relay in [16]. We perform dirty paper coding simultaneously for both the users instead of time sharing between the two users as was done in [13]. We derive outer bounds on the capacity region of the Gaussian interference channel with a cognitive relay using results on MIMO cognitive radio channel from [12] and using standard information theoretic inequalities. We also derive the degree of freedom (d.o.f.) region of the interference channel with cognitive relay. We show that we can achieve the full degrees of freedom of a two user no-interference channel for a large range of channel parameters.

Throughout the paper, we denote random variables by capital letters, their realizations by lower case and their alphabets by calligraphic letters (eg. $X, x$ and $\mathcal{X}$ respectively). We denote vectors of length $n$ with boldface letters (e.g. $\mathbf{x}^{n}$ ), and the $i^{\text {th }}$ element of a vector $\mathrm{x}^{n}$ by $x_{i}$. For any set $S, \bar{S}$ and $\mathrm{CH}(S)$ denote the closure and convex hull of $S$ respectively. For any vector or matrix $\mathbf{A}, \mathbf{A}^{\prime}$ denotes its transpose. $\operatorname{Tr}(\mathbf{A})$ denotes the trace of a matrix $\mathbf{A}$. We define the function $L: \mathbb{R}_{+} \rightarrow \mathbb{R}$ as $L(x)=\frac{1}{2} \log (1+x)$.

\section{System Model}

We study a Gaussian interference channel with two transmitters, two receivers and a cognitive relay. The system model is described in Figure 1. The interference channel is described by $\left(\mathcal{X}_{1}, \mathcal{X}_{2}, \mathcal{X}_{r}, \mathcal{Y}_{1}, \mathcal{Y}_{2}, p\left(y_{1}, y_{2} \mid x_{1}, x_{2}, x_{r}\right)\right)$, where $\mathcal{X}_{1}, \mathcal{X}_{2}, \mathcal{X}_{r}$ are the input alphabets associated with the two transmitters and the relay, $\mathcal{Y}_{1}, \mathcal{Y}_{2}$ are the two output alphabets. For the Gaussian channel, we assume that all the alphabets are the entire reals $\mathbb{R}$. Source $i, i=1,2$ has message 


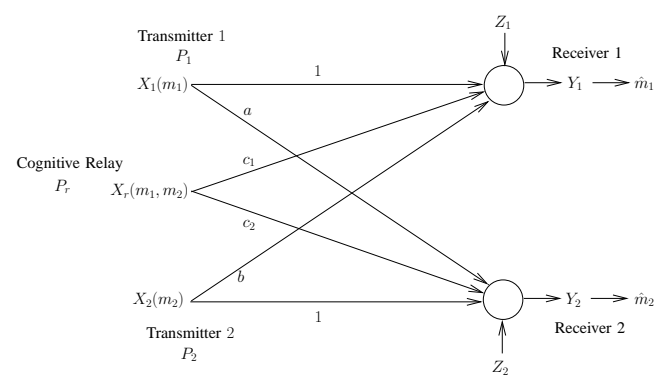

Fig. 1. System model for Gaussian Interference Channel with Cognitive Relay.

$m_{i} \in\left\{1, \ldots, 2^{n R_{i}}\right\}$ to be communicated to destination $i$ over $n$ channel uses. The relay has non-causal access to both the messages $m_{1}$ and $m_{2}$ and assists the two sources. Let $X_{1}, X_{2}, X_{r}$ and $Y_{1}, Y_{2}$ denote the random variables representing the respective channel inputs and outputs. Then, the inputoutput relationship can be represented by the system equations

$$
\begin{aligned}
& Y_{1}=X_{1}+b X_{2}+c_{1} X_{r}+Z_{1} \\
& Y_{2}=a X_{1}+X_{2}+c_{2} X_{r}+Z_{2},
\end{aligned}
$$

where $a, b, c_{1}$ and $c_{2}$ represent the channel gains as shown in Figure 1. $Z_{1}$ and $Z_{2}$ denote the additive noise which are i.i.d. Gaussian random variables distributed as $\mathcal{N}(0,1)$. The channel inputs must satisfy the following power constraints:

$$
\frac{1}{n} \sum_{i=1}^{n} E\left[X_{j, i}^{2}\right] \leq P_{j}, \quad j \in\{1,2, r\} .
$$

A $\left(2^{n R_{1}}, 2^{n R_{2}}, n, P e\right)$ code consists of message sets $M_{1}=$ $\left\{1, \ldots, 2^{n R_{1}}\right\}$ and $M_{2}=\left\{1, \ldots, 2^{n R_{2}}\right\}$, three encoding functions

$$
\begin{gathered}
f_{1}: M_{1} \rightarrow \mathcal{X}_{1}^{n}, \quad f_{2}: M_{2} \rightarrow \mathcal{X}_{2}^{n}, \\
f_{r}: M_{1} \times M_{2} \rightarrow \mathcal{X}_{r}^{n},
\end{gathered}
$$

and two decoding functions

$$
g_{1}: \mathcal{Y}_{1}^{n} \rightarrow M_{1}, \quad g_{2}: \mathcal{Y}_{2}^{n} \rightarrow M_{2},
$$

such that the transmitted codewords $\mathbf{X}_{1}^{n}, \mathbf{X}_{2}^{n}$ and $\mathbf{X}_{r}^{n}$ satisfy the power constraints given by (2) and an error probability $\leq P e=\max \left(P_{e, 1}, P_{e, 2}\right)$. For $t=1,2$, we have

$$
P_{e, t}=\frac{1}{2^{n\left(R_{1}+R_{2}\right)}} \sum_{\left(m_{1}, m_{2}\right)} \operatorname{Pr}\left[g\left(\mathbf{Y}_{t}^{n}\right) \neq m_{t} \mid\left(m_{1}, m_{2}\right) \text { sent }\right] \text {. }
$$

A rate pair $\left(R_{1}, R_{2}\right)$ is achievable if there exists a sequence of $\left(2^{n R_{1}}, 2^{n R_{2}}, n, P e^{(n)}\right)$ codes such that $P e^{(n)} \rightarrow 0$ as $n \rightarrow \infty$. The capacity region of the interference channel with cognitive relay is then the set of all rate pairs $\left(R_{1}, R_{2}\right)$ that are achievable, and is denoted by $\mathcal{C}_{I C}$. The d.o.f. region of the Gaussian interference channel with cognitive relay $\mathcal{D}$ is defined as

$$
\mathcal{D}=\left\{\begin{array}{l}
\left(d_{1}, d_{2}\right) \in \mathbb{R}_{+}^{2}: \forall w \in \mathbb{R}_{+}, \\
w d_{1}+d_{2} \leq \lim \sup _{P_{1}+P_{2}+P_{r} \rightarrow \infty} \\
\sup _{\left(R_{1}, R_{2}\right) \in \mathcal{C}_{I C} \frac{w R_{1}+R_{2}}{\log \left(P_{1}+P_{2}+P_{r}\right)}}
\end{array}\right\} .
$$

\section{MAIN RESUltS}

Let $\mathcal{P}$ denote the set of $\left(P_{11}, P_{12}, P_{21}, P_{22}, P_{r 11}, P_{r 12}, P_{r 21}, P_{r 22}, P_{r 3}, P_{r 4}\right)$ described by

$$
\left\{\begin{array}{c}
\left(\begin{array}{c}
P_{11}, P_{12}, P_{21}, P_{22}, P_{r 11}, P_{r 12}, \\
P_{r 21}, P_{r 22}, P_{r 3}, P_{r 4}
\end{array}\right): \\
P_{11}+P_{12}=P_{1} \\
P_{21}+P_{22}=P_{2} \\
P_{r 11}+P_{r 12}+P_{r 21}+P_{r 22}+P_{r 3}+P_{r 4}=P_{r}
\end{array}\right\} .
$$

Let $P^{*} \in \mathcal{P}$. Let $\alpha_{1}, \alpha_{2}, \beta_{1}, \beta_{2} \in\{-1,1\}$. We denote $r_{11}, r_{12}, r_{21}, r_{22}$ as follows :

$$
\begin{aligned}
& r_{1 j}=\left(\sqrt{P_{1 j}}+\alpha_{j} c_{1} \sqrt{P_{r 1 j}}\right)^{2}, \quad j \in\{1,2\}, \\
& r_{2 j}=\left(b \sqrt{P_{2 j}}+\beta_{j} c_{1} \sqrt{P_{r 2 j}}\right)^{2}, \quad j \in\{1,2\} .
\end{aligned}
$$

Let $\mathcal{R}_{i 1}^{P^{*}}\left(\alpha_{1}, \alpha_{2}, \beta_{1}, \beta_{2}\right)$ denote the set described by

$$
\left\{\begin{array}{l}
\left(R_{11}, R_{12}, R_{21}\right): R_{11} \geq 0, R_{12} \geq 0, R_{21} \geq 0 \\
R_{11} \leq L\left(\frac{r_{11}}{1+r_{22}+c_{1}^{2}\left(P_{r 3}+P_{r 4}\right)}\right) \\
R_{12} \leq L\left(\frac{r_{12}}{1+r_{22}+c_{1}^{2}\left(P_{r 3}+P_{r 4}\right)}\right) \\
R_{21} \leq L\left(\frac{r_{21}}{1+r_{22}+c_{1}^{2}\left(P_{r 3}+P_{r 4}\right)}\right) \\
R_{11}+R_{12} \leq L\left(\frac{r_{11}+r_{12}}{1+r_{22}+c_{1}^{2}\left(P_{r 3}+P_{r 4}\right)}\right) \\
R_{11}+R_{21} \leq L\left(\frac{r_{11}+r_{21}}{1+r_{22}+c_{1}^{2}\left(P_{r 3}+P_{r 4}\right)}\right) \\
R_{12}+R_{21} \leq L\left(\frac{r_{12}+r_{21}}{1+r_{22}+c_{1}^{2}\left(P_{r 3}+P_{r 4}\right)}\right) \\
R_{11}+R_{12}+R_{21} \leq L\left(\frac{r_{11}+r_{12}+r_{21}}{1+r_{22}+c_{1}^{2}\left(P_{r 3}+P_{r 4}\right)}\right)
\end{array}\right\} .
$$

We denote $s_{11}, s_{12}, s_{21}, s_{22}$ as follows :

$$
\begin{array}{cl}
s_{1 j}=\left(a \sqrt{P_{1 j}}+\alpha_{j} c_{2} \sqrt{P_{r 1 j}}\right)^{2}, & j \in\{1,2\}, \\
s_{2 j}=\left(\sqrt{P_{2 j}}+\beta_{j} c_{2} \sqrt{P_{r 2 j}}\right)^{2}, \quad j \in\{1,2\} .
\end{array}
$$

$\mathcal{R}_{i 2}^{P^{*}}\left(\alpha_{1}, \alpha_{2}, \beta_{1}, \beta_{2}\right)$ denotes the set described by

$$
\left\{\begin{array}{l}
\left(R_{12}, R_{21}, R_{22}\right): R_{12} \geq 0, R_{21} \geq 0, R_{22} \geq 0 \\
R_{12} \leq L\left(\frac{s_{12}}{1+s_{11}+c_{2}^{2}\left(P_{r 3}+P_{r 4}\right)}\right) \\
R_{21} \leq L\left(\frac{s_{21}}{1+s_{11}+c_{2}^{2}\left(P_{r 3}+P_{r 4}\right)}\right) \\
R_{22} \leq L\left(\frac{s_{22}}{1+s_{11}+c_{2}^{2}\left(P_{r 3}+P_{r 4}\right)}\right) \\
R_{12}+R_{21} \leq L\left(\frac{s_{12}+s_{21}}{1+s_{11}+c_{2}^{2}\left(P_{r 3}+P_{r 4}\right)}\right) \\
R_{12}+R_{22} \leq L\left(\frac{s_{12}+s_{22}}{1+s_{11}+c_{2}^{2}\left(P_{r 3}+P_{r 4}\right)}\right) \\
R_{21}+R_{22} \leq L\left(\frac{s_{21}+s_{22}}{1+s_{11}+c_{2}^{2}\left(P_{r 3}+P_{r 4}\right)}\right) \\
R_{12}+R_{21}+R_{22} \leq\left(\frac{s_{12}+s_{21}+s_{22}}{1+s_{11}+c_{2}^{2}\left(P_{r 3}+P_{r 4}\right)}\right)
\end{array}\right\} .
$$


Let $\bar{\alpha}=\left(\alpha_{1}, \alpha_{2}\right)$ and $\bar{\beta}=\left(\beta_{1}, \beta_{2}\right)$. Let $\mathcal{R}_{i n, 1}^{P^{*}}(\bar{\alpha}, \bar{\beta})$ and $\mathcal{R}_{i n, 2}^{P^{*}}(\bar{\alpha}, \bar{\beta})$ be the set of rate pairs $\left(R_{1}, R_{2}\right)$ described by

$$
\begin{gathered}
\mathcal{R}_{i n, 1}^{P^{*}}(\bar{\alpha}, \bar{\beta})=\left\{\begin{array}{l}
\left(R_{1}, R_{2}\right): R_{1} \geq 0, R_{2} \geq 0 \\
R_{1}=R_{11}+R_{12}+R_{13} \\
R_{2}=R_{21}+R_{22}+R_{23} \\
\left(R_{11}, R_{12}, R_{21}\right) \in \mathcal{R}_{i 1}^{P^{*}}(\bar{\alpha}, \bar{\beta}) \\
\left(R_{12}, R_{21}, R_{22}\right) \in \mathcal{R}_{i 2}^{P^{*}}(\bar{\alpha}, \bar{\beta}) \\
R_{13} \leq L\left(\frac{c_{1}^{2} P_{3}}{1+c_{1}^{2} r_{r 4}}\right) \\
R_{23} \leq L\left(c_{2}^{2} P_{r 4}\right)
\end{array}\right\}, \\
\mathcal{R}_{i n, 2}^{P^{*}}(\bar{\alpha}, \bar{\beta})=\left\{\begin{array}{l}
\left(R_{1}, R_{2}\right): R_{1} \geq 0, R_{2} \geq 0 \\
R_{1}=R_{11}+R_{12}+R_{13} \\
R_{2}=R_{21}+R_{22}+R_{23} \\
\left(R_{11}, R_{12}, R_{21}\right) \in \mathcal{R}_{i 1}^{P^{*}}(\bar{\alpha}, \bar{\beta}) \\
\left(R_{12}, R_{21}, R_{22}\right) \in \mathcal{R}_{i 2}^{P^{*}}(\bar{\alpha}, \bar{\beta}) \\
R_{13} \leq L\left(c_{1}^{2} P_{r 3}\right) \\
R_{23} \leq L\left(\frac{c_{2}^{2} P_{r 4}}{1+c_{2}^{2} P_{r 3}}\right)
\end{array}\right\} .
\end{gathered}
$$

Let $\mathcal{R}_{i n}$ be the set of rate pairs described by

$$
\mathcal{R}_{i n}=\overline{\mathrm{CH}\left(\bigcup_{P^{*} \in \mathcal{P}} \bigcup_{\bar{\alpha}, \bar{\beta}}\left(\mathcal{R}_{i n, 1}^{P^{*}}(\bar{\alpha}, \bar{\beta}) \cup \mathcal{R}_{i n, 2}^{P^{*}}(\bar{\alpha}, \bar{\beta})\right)\right)} .
$$

Then, the following theorem describes an achievable region for the Gaussian interference channel with cognitive relay.

Theorem 1: The capacity region of the Gaussian interference channel with cognitive relay $\mathcal{C}_{I C}$ satisfies

$$
\mathcal{R}_{\text {in }} \subseteq \mathcal{C}_{I C} .
$$

The outline of the proof of Theorem 1 is described in Section IV. The coding scheme used to achieve the region given by $\mathcal{R}_{i n}$ is a combination of Han-Kobayashi coding scheme for an interference channel [14] and Costa's dirty paper coding [15].

Let $\gamma>0$ be any positive real number. We define the following $3 \times 1$ matrices:

$$
\begin{array}{lll}
\mathbf{G}_{1 \gamma}=\left[\begin{array}{lll}
1 & \frac{c_{1}}{\sqrt{\gamma}} & \frac{b}{\sqrt{\gamma}}
\end{array}\right], & \mathbf{H}_{1 \gamma}=\left[\begin{array}{lll}
0 & \frac{c_{2}}{\sqrt{\gamma}} & \frac{1}{\sqrt{\gamma}}
\end{array}\right], \\
\mathbf{G}_{2 \gamma}=\left[\begin{array}{llll}
\frac{1}{\sqrt{\gamma}} & \frac{c_{1}}{\sqrt{\gamma}} & 0
\end{array}\right], & \mathbf{H}_{2 \gamma}=\left[\begin{array}{lll}
\frac{a}{\sqrt{\gamma}} & \frac{c_{2}}{\sqrt{\gamma}} & 1
\end{array}\right] .
\end{array}
$$

Consider the two 2-user Gaussian MIMO broadcast channels given in Figures 2 and 3 with three transmit antennas and one antenna at each receiver. We denote the two broadcast

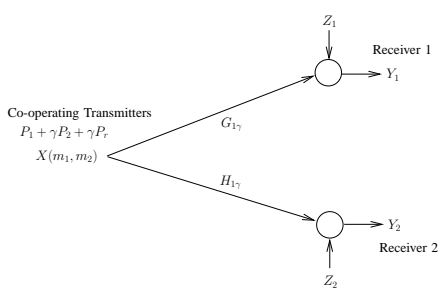

Fig. 2. Broadcast Channel 1.

channels as $B C_{1}^{\gamma}$ and $B C_{2}^{\gamma}$ respectively. Let their capacity regions be denoted by $\mathcal{C}_{B C, 1}^{\gamma}$ and $\mathcal{C}_{B C, 2}^{\gamma}$ respectively. $\mathcal{R}_{B C, 1}^{\gamma}$

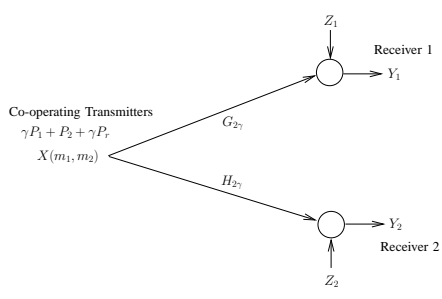

Fig. 3. Broadcast Channel 2.

represents the closure of the convex hull of the set of rate pairs described by

$$
\left\{\begin{array}{l}
\left(R_{1}, R_{2}\right): R_{1} \geq 0, R_{2} \geq 0 \\
R_{1} \leq L\left(\frac{\mathbf{G}_{1 \gamma} \boldsymbol{\Sigma}_{1} \mathbf{G}_{1 \gamma}^{\prime}}{1+\mathbf{G}_{1 \gamma}\left(\boldsymbol{\Sigma}_{1}+\boldsymbol{\Sigma}_{2}\right) \mathbf{G}_{1 \gamma}^{\prime}}\right) \\
R_{2} \leq L\left(\mathbf{H}_{1 \gamma} \boldsymbol{\Sigma}_{2} \mathbf{H}_{1 \gamma}^{\prime}\right) \\
\boldsymbol{\Sigma}_{1} \succeq \mathbf{0}, \boldsymbol{\Sigma}_{2} \succeq \mathbf{0} \\
\operatorname{Tr}\left(\boldsymbol{\Sigma}_{1}+\boldsymbol{\Sigma}_{2}\right) \leq P_{1}+\gamma P_{2}+\gamma P_{r}
\end{array}\right\} .
$$

$\mathcal{R}_{B C, 2}^{\gamma}$ represents the closure of the convex hull of the set of rate pairs described by

$$
\left\{\begin{array}{l}
\left(R_{1}, R_{2}\right): R_{1} \geq 0, R_{2} \geq 0 \\
R_{1} \leq L\left(\mathbf{G}_{2 \gamma} \boldsymbol{\Sigma}_{1} \mathbf{G}_{2 \gamma}^{\prime}\right) \\
R_{2} \leq L\left(\frac{\mathbf{H}_{2 \gamma} \boldsymbol{\Sigma}_{2} \mathbf{H}_{2 \gamma}^{\prime}}{1+\mathbf{H}_{2 \gamma}\left(\boldsymbol{\Sigma}_{1}+\boldsymbol{\Sigma}_{2}\right) \mathbf{H}_{2 \gamma}^{\prime}}\right) \\
\boldsymbol{\Sigma}_{1} \succeq \mathbf{0}, \quad \boldsymbol{\Sigma}_{2} \succeq \mathbf{0} \\
\operatorname{Tr}\left(\boldsymbol{\Sigma}_{1}+\boldsymbol{\Sigma}_{2}\right) \leq \gamma P_{1}+P_{2}+\gamma P_{r}
\end{array}\right\} .
$$

Then, we have the following lemma.

Lemma 1: For any $\mu \geq 1$, we have

$$
\begin{gathered}
\max _{\left(R_{1}, R_{2}\right) \in \mathcal{R}_{B C, 1}^{\gamma}} \mu R_{1}+R_{2}=\max _{\left(R_{1} m R_{2}\right) \in \mathcal{C}_{B C, 1}^{\gamma}} \mu R_{1}+R_{2} \\
\max _{\left(R_{1}, R_{2}\right) \in \mathcal{R}_{B C, 2}^{\gamma}} R_{1}+\mu R_{2}=
\end{gathered}
$$

The proof of the lemma follows directly from the results of [17] and is omitted here. The following theorem describes an outer bound on the capacity region of the Gaussian interference channel with cognitive relay.

Theorem 2: Let $\mu \geq 1$. The capacity region of the Gaussian interference channel with cognitive relay, $\mathcal{C}_{I C}$ satisfies

$$
\begin{gathered}
\max _{\left(R_{1}, R_{2}\right) \in \mathcal{C}_{I C}} \mu R_{1}+R_{2} \leq \min _{\gamma>0} \max _{\left(R_{1}, R_{2}\right) \in \mathcal{R}_{B C, 1}^{\gamma}} \mu R_{1}+R_{2} \\
\max _{\left(R_{1}, R_{2}\right) \in \mathcal{C}_{I C}} R_{1}+\mu R_{2} \leq \min _{\gamma>0} \max _{\left(R_{1}, R_{2}\right) \in \mathcal{R}_{B C, 2}^{\gamma}} R_{1}+\mu R_{2} .
\end{gathered}
$$

An outline of the proof of Theorem 2 is described in Section V. It is to be noted that the outer bound is not just the capacity region of the broadcast channel obtained by allowing all the transmitters to co-operate. In fact, the broadcast channel considered in Figures 2 and 3 have some channel gains that are set to zero. The outer bound is obtained by considering the outer bound of a MIMO cognitive channel [12] that results from partial transmitter co-operation.

Let $\rho_{1}, \rho_{2} \in[-1,1]$. Let $\mathbf{A}\left(\rho_{1}, \rho_{2}\right)$ be given by

$$
A\left(\rho_{1}, \rho_{2}\right)=\left(\begin{array}{ccc}
P_{1} & 0 & \rho_{1} \sqrt{P_{1} P_{r}} \\
0 & P_{2} & \rho_{2} \sqrt{P_{2} P_{r}} \\
\rho_{1} \sqrt{P_{1} P_{r}} & \rho_{2} \sqrt{P_{2} P_{r}} & P_{r}
\end{array}\right) .
$$


We define the functions $F_{1}\left(\rho_{1}, \rho_{2}\right)$ and $F_{2}\left(\rho_{1}, \rho_{2}\right)$ as

$$
\begin{aligned}
& F_{1}\left(\rho_{1}, \rho_{2}\right)=L\left(P_{1}+c_{1}^{2} P_{r}\left(1-\rho_{2}^{2}\right)+2 c_{1} \rho_{1} \sqrt{P_{1} P_{r}}\right) \\
& F_{2}\left(\rho_{1}, \rho_{2}\right)=L\left(P_{2}+c_{2}^{2} P_{r}\left(1-\rho_{1}^{2}\right)+2 c_{2} \rho_{2} \sqrt{P_{2} P_{r}}\right) .
\end{aligned}
$$

The following theorem describes another outer bound on $\mathcal{C}_{I C}$.

Theorem 3: Let $\left(R_{1}, R_{2}\right) \in \mathcal{C}_{I C}$. Then for any $0 \leq \mu<\infty$, we have

$$
\begin{aligned}
\mu R_{1}+R_{2} \leq & \max _{\rho_{1}, \rho_{2} \in[-1,1]} \mu F_{1}\left(\rho_{1}, \rho_{2}\right)+F_{2}\left(\rho_{1}, \rho_{2}\right) \\
& \text { such that } A\left(\rho_{1}, \rho_{2}\right) \succeq 0 .
\end{aligned}
$$

The proof of the theorem follows from a series of basic information theoretic inequalities and is omitted here. The following theorem characterizes the d.o.f. region of the Gaussian interference channel with cognitive relay.

Theorem 4: If $c_{1} a \neq c_{2}$ and $c_{2} b \neq c_{1}$, the d.o.f. region of the Gaussian interference channel with cognitive relay is

$$
\mathcal{D}_{1}=\left\{\begin{array}{l}
\left(d_{1}, d_{2}\right) \in \mathbb{R}_{+}^{2}: \\
d_{1} \leq 1, \quad d_{2} \leq 1
\end{array}\right\}
$$

If $c_{1} a=c_{2}$ and/or $c_{2} b=c_{1}$, then the d.o.f. region of the cognitive relay is given by

$$
\mathcal{D}_{2}=\left\{\begin{array}{l}
\left(d_{1}, d_{2}\right) \in \mathbb{R}_{+}^{2}: \\
d_{1}+d_{2} \leq 1
\end{array}\right\} .
$$

Proof: We first consider the case when $c_{1} a \neq c_{2}$ and $c_{2} b \neq c_{1}$. We describe an outer bound on the d.o.f. region. We allow all the three transmitters to co-operate and obtain a two user broadcast channel with 3 antennas at the transmitter and 1 antenna at each receiver. The d.o.f. region of the broadcast channel is equal to the region described by (26). Hence, the region described by $\mathcal{D}_{1}$ is an outer bound on the d.o.f. region of the Gaussian interference channel with cognitive relay.

We now show that the d.o.f. region $\mathcal{D}_{1}$ is achievable by interference cancelation. For $i=1,2$, transmitter $i$ chooses its transmit codeword $X_{i}$ according to the distribution $X_{i} \backsim$ $\mathcal{N}\left(0, Q_{i}\right), Q_{i} \leq P_{i}$. The relay transmits $X_{r}=\lambda_{1} X_{1}+\lambda_{2} X_{2}$. Hence, we must have $\lambda_{1}^{2} Q_{1}+\lambda_{2}^{2} Q_{2} \leq P_{r}$. We choose $\lambda_{2}=$ $-\frac{b}{c_{1}}$ and $\lambda_{1}=-\frac{a}{c_{2}}$, to cancel out the interference at each receiver. To satisfy the power constraints, we choose $Q_{i}=$ $\min \left(\frac{P_{r}}{2 \lambda_{i}^{2}}, P_{i}\right), i=1,2$. We then achieve the point $\left(d_{1}, d_{2}\right)=$ $(1,1)$. Hence, the region $\mathcal{D}_{1}$ is achievable.

Next, we consider the case when $c_{1} a=c_{2}$ and/or $c_{2} b=c_{1}$. The region given by $\mathcal{D}_{2}$ is achievable by time sharing. When $c_{1} a=c_{2}$, using arguments similar to those used in [1], we can show that receiver 2 can decode both the messages $m_{1}$ and $m_{2}$ successfully, and that is the optimal strategy for receiver 2. Hence, $d_{1}+d_{2} \leq 1$ is an upper bound on the d.o.f. region. The proof is similar for the case when $c_{2} b=c_{1}$.

\section{Achievable Region : Theorem 1}

Outline of Proof of Theorem 1 : We fix a $P^{*} \in \mathcal{P}$ where $\mathcal{P}$ is described in (7). We also fix $\alpha_{1}, \alpha_{2}, \beta_{1}, \beta_{2} \in\{-1,1\}$. We show that $\mathcal{R}_{i n, 1}^{P^{*}}(\bar{\alpha}, \bar{\beta})$ is achievable. We assume that $P_{11}, P_{12}, P_{21}, P_{22}>0$. The proof for the case when some of $P_{11}, P_{12}, P_{21}, P_{22}$ are equal to zero is almost identical to the one presented here and is hence omitted.

For $i=1,2$, source $i$ splits its message $m_{i} \in\left\{1, \ldots, 2^{n R_{i}}\right\}$ into 3 independent parts $\left(m_{i 1}, m_{i 2}, m_{i 3}\right) \in\left\{1, \ldots, 2^{n R_{i 1}}\right\} \times$ $\left\{1, \ldots, 2^{n R_{i 2}}\right\} \times\left\{1, \ldots, 2^{n R_{i 3}}\right\}$ such that $R_{i 1}+R_{i 2}+R_{i 3}=$ $R_{i}$.

Encoding Scheme : For $i=1,2$, transmitter $i$ encodes message $m_{i 1}$ into $\mathbf{X}_{i 1}^{n}$, such that $p\left(\mathbf{x}_{i 1}^{n}\right)=\Pi_{j=1}^{n} P\left(x_{i 1, j}\right)$, and $X_{i 1, j} \backsim \mathcal{N}\left(0, P_{i 1}\right)$. Message $m_{i 2}$ is then encoded into $\mathbf{X}_{i 2}^{n}$, such that $p\left(\mathbf{x}_{i 2}^{n}\right)=\prod_{j=1}^{n} P\left(x_{i 2, j}\right)$, and $X_{i 2, j} \backsim \mathcal{N}\left(0, P_{i 2}\right)$. Transmitter $i$ transmits $\mathbf{X}_{i}^{n}=\mathbf{X}_{i 1}^{n}+\mathbf{X}_{i 2}^{n}$.

The relay encodes message $\left(m_{11}, m_{12}\right)$ into $\mathbf{X}_{r 1}^{n}=\alpha_{1} \sqrt{\left(P_{r 11} / P_{11}\right)} \mathbf{X}_{11}^{n}+\alpha_{2} \sqrt{\left(P_{r 12} / P_{12}\right)} \mathbf{X}_{12}^{n}$, and message $\left(m_{21}, m_{22}\right)$ into $\mathbf{X}_{r 2}^{n}=\beta_{1} \sqrt{\left(P_{r 21} / P_{21}\right)} \mathbf{X}_{21}^{n}+$ $\beta_{2} \sqrt{\left(P_{r 22} / P_{22}\right)} \mathbf{X}_{22}^{n}$. The relay node encodes message $m_{13}$ into $\mathbf{X}_{r 3}^{n}$ treating $\left(b+c_{1} \beta_{2} \sqrt{\left(P_{r 22} / P_{22}\right)}\right) \mathbf{X}_{22}^{n}$ as non-causally known interference at receiver 1 . That is, $\mathbf{X}_{r 3}^{n}$ is formed using Costa's dirty paper coding [15], and is distributed as $p\left(\mathbf{x}_{r 3}^{n}\right)=\Pi_{i=1}^{n} P\left(x_{r 3, i}\right)$ and $X_{r 3, i} \backsim \mathcal{N}\left(0, P_{r 3}\right)$. Finally, the relay encodes message $m_{23}$ into $\mathbf{X}_{r 4}^{n}$ treating $\left(a+c_{2} \alpha_{1} \sqrt{\left(P_{r 11} / P_{11}\right)}\right) \mathbf{X}_{11}^{n}+c_{2} \mathbf{X}_{r 3}^{n}$ as non-causally known interference at receiver $2 . \mathbf{X}_{r 4}^{n}$ is distributed as $p\left(\mathbf{x}_{r 4}^{n}\right)=\prod_{i=1}^{n} P\left(x_{r 4, i}\right)$ and $X_{r 4, i} \backsim \mathcal{N}\left(0, P_{r 4}\right)$. The relay transmits $\mathbf{X}_{r}^{n}=\mathbf{X}_{r 1}^{n}+\mathbf{X}_{r 2}^{n}+\mathbf{X}_{r 3}^{n}+\mathbf{X}_{r 4}^{n}$. It is to be noted that this coding scheme uses the result that the capacity region of a Gaussian broadcast channel with additive state known non-causally at the transmitter is the same as the capacity region of the same broadcast channel with no state [18].

Decoding : Receiver 1 decodes $\left(m_{11}, m_{12}, m_{21}\right)$ jointly by treating $\left(b+c_{1} \beta_{2} \sqrt{\left(P_{r 22} / P_{22}\right)}\right) \mathbf{X}_{22}^{n}+c_{1}\left(\mathbf{X}_{r 3}^{n}+\mathbf{X}_{r 4}^{n}\right)+\mathbf{Z}_{1}^{n}$ as Gaussian noise. Hence, $\left(m_{11}, m_{12}, m_{21}\right)$ can be successfully decoded at receiver 1 if $\left(R_{11}, R_{12}, R_{21}\right) \in \mathcal{R}_{i 1}^{P^{*}}(\bar{\alpha}, \bar{\beta})$. Receiver 1 then decodes message $m_{13}$ by treating $c_{1} \mathbf{X}_{r 4}^{n}+\mathbf{Z}_{1}^{n}$ as Gaussian noise.

Receiver 2 decodes $\left(m_{12}, m_{21}, m_{22}\right)$ jointly by treating $(a+$ $\left.c_{2} \alpha_{1} \sqrt{\left(P_{r 11} / P_{11}\right)}\right) \mathbf{X}_{11}^{n}+c_{2}\left(\mathbf{X}_{r 3}^{n}+\mathbf{X}_{r 4}^{n}\right)+\mathbf{Z}_{2}^{n}$ as Gaussian noise. Hence, $\left(m_{12}, m_{21}, m_{22}\right)$ can be successfully decoded at receiver 2 if $\left(R_{12}, R_{21}, R_{22}\right) \in \mathcal{R}_{i 2}^{P^{*}}(\bar{\alpha}, \bar{\beta})$. Finally, message $m_{23}$ is decoded by treating $\mathbf{Z}_{2}^{n}$ as noise.

Hence, it follows that $\mathcal{R}_{i n, 1}^{P^{*}}(\alpha, \beta)$ is achievable. Similarly, $\mathcal{R}_{i n, 2}^{P^{*}}(\alpha, \beta)$ is also achievable. Hence, the proof follows.

Remark 1: There are two main differences between the achievable region presented in this paper and the one given in [13]. The first one is that, we incorporate message splitting and partial interference cancelation at the receiver. This strategy is motivated by the Han-Kobayashi coding scheme for the general interference channel [14]. The second major difference is, we perform dirty paper coding for both the users simultaneously and time share the order in which we perform dirty paper coding. In [13], the authors perform dirty paper coding for only one user at a time and time share between the two dirty paper coding regions. 


\section{Outer Bound : Theorem 2}

Outline of Proof of Theorem 2 : The outer bound is obtained by allowing transmitter co-operation. We allow transmitter 2 to fully co-operate with the relay. This is done by providing transmitter 2 with message $m_{1}$ non-causally. This reduces the channel to a Gaussian MIMO cognitive channel studied in [12]. Let the capacity region of the corresponding MIMO cognitive channel be denoted by $\mathcal{C}_{M C C, 1}$. Then, for any $\mu \geq 1$, it is shown in [12, Theorem 3.2 and Lemma 5.6] that

$$
\max _{\left(R_{1}, R_{2}\right) \in \mathcal{C}_{M C C, 1}} \mu R_{1}+R_{2} \leq \min _{\gamma>0} \max _{\left(R_{1}, R_{2}\right) \in \mathcal{R}_{B C, 1}^{\gamma}} \mu R_{1}+R_{2} .
$$

It follows that for any $\mu \geq 1$,

$$
\max _{\left(R_{1}, R_{2}\right) \in \mathcal{C}_{I C}} \mu R_{1}+R_{2} \leq \min _{\gamma>0} \max _{\left(R_{1}, R_{2}\right) \in \mathcal{R}_{B C, 1}^{\gamma}} \mu R_{1}+R_{2} .
$$

By allowing transmitter 1 to co-operate fully with the relay node, we obtain the other bound. That is, for any $\mu \geq 1$,

$$
\max _{\left(R_{1}, R_{2}\right) \in \mathcal{C}_{I C}} R_{1}+\mu R_{2} \leq \min _{\gamma>0} \max _{\left(R_{1}, R_{2}\right) \in \mathcal{R}_{B C, 2}^{\gamma}} R_{1}+\mu R_{2} .
$$

Remark 2: It is to be noted that the outer bound is not obtained by merely letting all the transmitters co-operate with a sum power constraint. In the broadcast channel in Figures 2 and 3 , it can be seen that one of the channel gains is made zero. Also, the outer bound is obtained by minimizing over a series of broadcast channel with different sum power constraints and channel gains. The outer bound obtained is in general not tight, even with respect to the cognitive radio channel [10][12], because, the non cognitive transmitter in the cognitive radio channel cannot transmit any information with respect to the message of the other transmitter.

\section{Numerical Results}

In this section, we provide some numerical results on the capacity region of the two user Gaussian interference channel with a cognitive relay. We consider an example system, where $a=b=2, c_{1}=1.5, c_{2}=0.75$. We take all power constraints to be equal to 10 (i.e., $P_{1}=P_{2}=P_{r}=10$ ). Figure 4 plots the achievable region $\mathcal{R}_{i n}$ described in (14), and the outer bounds in Theorem 2 and Lemma 2. The plot shows the performance improvements over the achievable region by [13] and the gap between the achievable region and the outer bounds.

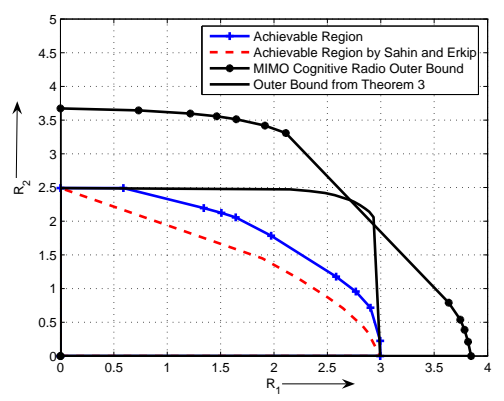

Fig. 4. Plot of Achievable region and Outer bound for Interference channel with Cognitive Helper

\section{CONCLUSIONS}

In this paper, we derived a new achievable region for the two user Gaussian interference channel with a cognitive relay. The achievable region is a generalization of the region given in [13]. In Theorems 2 and 3, we derive outer bounds on the capacity region of the interference channel with cognitive relay. We also derive the d.o.f. region of the channel setting and show that we can achieve the full degrees of freedom of a two user no-interference channel for a large range of channel parameters.

\section{REFERENCES}

[1] H. Sato, "The capacity of the Gaussian interference channel under strong interference," IEEE Trans. Inform. Theory, vol. 27, no. 6, pp. 786-788, Nov. 1981.

[2] M. H. M. Costa and A. A. E. Gamal, "The capacity region of the discrete memoryless interference channel with strong interference," IEEE Trans. Inform. Theory, vol. 33, no. 5, pp. 710-711, Sept. 1987.

[3] A. A. E. Gamal and M. H. M. Costa, "The capacity region of a class of determinstic interference channels," IEEE Trans. Inform. Theory, vol. 28, no. 2, pp. 343-346, March 1982.

[4] R. Benzel, "The capacity region of a class of discrete additive degraded interference channels," IEEE Trans. Inform. Theory, vol. 25, no. 2, pp. 228-231, March 1979.

[5] R. H. Etkin, D. N. C. Tse, and H. Wang, "Gaussian interference channel capacity to within one bit," submitted to IEEE Trans. Inform. Theory, Feb 2007.

[6] X. Shang, G. Kramer, and B. Chen, "A new outer bound and the noisyinterference sum-rate capacity for Gaussian interference channels," submitted to IEEE Trans. Inform. Theory, Dec. 2007.

[7] A. A. Motahari and A. K. Khandani, "Capacity bounds for the gaussian interference channel," Library and Archives Canada Technical Report UW-ECE 2007 -26, Tech. Rep., Aug. 2007.

[8] N. Devroye, P. Mitran, and V. Tarokh, "Achievable rates in cognitive radio channels," IEEE Trans. Inform. Theory, vol. 52, no. 5, pp. 18131827, May 2006.

[9] I. Marić, A. Goldsmith, G. Kramer, and S. Shamai, "On the capacity of interference channel with one co-operating transmitter," European Trans. on Telecomm., to appear in 2008.

[10] W. Wu, S. Vishwanath, and A. Arapostathis, "Capacity of a class of cognitive radio channels: Interference channels with degraded message sets," IEEE Trans. Inform. Theory, vol. 53, no. 11, pp. 4391-4399, Nov. 2007.

[11] A. Jovicic and P. Viswanath, "Cognitive radio: An information-theoretic perspective," to appear in IEEE Trans. Inform. Theory. Preprint available at http://arxiv.org/abs/cs.IT/0604107.

[12] S. Sridharan and S. Vishwanath, "On the capacity of a class of MIMO cognitive radios," to appear in Journal on Selected Topics in Signal Processing, 2007. Preprint available at http://arxiv.org/abs/0711.4792v2.

[13] O. Sahin and E. Erkip, "On achievable rates for interference relay channel with interference cancelation," in Proc. of Forty First Annual Asilomar Conf. on Signals, Systems and Computers, Pacific Grove, California, vol. Nov., 2007.

[14] T. S. Han and K. Kobayashi, "A new achievable rate region for the interference channel," IEEE Trans. Inform. Theory, vol. 27, no. 1, pp. 49-60, Jan. 1981.

[15] M. Costa, "Writing on dirty paper (corresp.)," IEEE Trans. Inform. Theory, vol. 29, no. 3, pp. 439-441, May 1983.

[16] O. Sahin and E. Erkip, "Achievable rates for the Gaussian interference relay channel," in Proc. of 2007 GLOBECOM Communication Theory Symposium, Washington D.C., Nov. 2007.

[17] H. Weingarten, Y. Steinberg, and S. Shamai, "The capacity region of the Gaussian multiple-input multiple-output broadcast channel," IEEE Trans. Inform. Theory, vol. 52, no. 9, pp. 3936-3964, Sept. 2006.

[18] Y. Steinberg and S. Shamai, "Achievable rates for the broadcast channel with states known at the transmitter," in Proc. of Intl. Symp. of Inform. Theory, Sep. 2005, 2005. 